\title{
Pleomorphic lobular carcinoma: is it more similar to a classic lobular cancer or to a high-grade ductal cancer?
}

\section{Leopoldo Costarelli \\ Domenico Campagna \\ Alessandra Ascarelli \\ Francesco Cavaliere \\ Maria Helena Colavito \\ Tatiana Ponzani \\ Laura Broglia \\ Massimo La Pinta \\ Elena Manna \\ Lucio Fortunato}

Breast Unit, San Giovanni-Addolorata Hospital, Rome, Italy
Correspondence: Lucio Fortunato Breast Unit, Azienda Ospedaliera San Giovanni Addolorata, Via Amba Aradam 9, 00187 Rome, Italy

Tel +39067 7056762

Fax +39067 7055549

Email Ifortunato@hsangiovanni.roma.it
This article was published in the following Dove Press journal: Breast Cancer - Targets and Therapy

Background: Pleomorphic invasive lobular carcinoma (P-ILC) is an uncommon variety of invasive lobular carcinoma with aggressive clinical features. Little is described in the literature regarding this topic.

Materials and methods: We reviewed our experiences from 2010 to 2015 and compared 40 patients with P-ILC, 126 patients with classic-ILC (C-ILC) and 574 cases of high-grade invasive ductal carcinoma (HG-IDC). We studied the histologic and immunohistochemical features, clinical presentation and surgical treatment.

Results: P-ILC is diagnosed at the same age and tumor diameter as those of the other two histologic types. It is associated more frequently with multiple lymph node metastases and high proliferative index, and HER $2 /$ neu is amplified in $10 \%$ of cases. In spite of sharing some histologic characteristics with C-ILC (same growth pattern, loss of E-cadherin expression, same genetic pathway), its clinical and pathologic features define an autonomous entity. Its surgical treatment is similar to those of C-ILC and HG-IDC.

Conclusion: This is the first review comparing these three pathologic entities. Our findings may be useful in understanding this variety of invasive lobular carcinoma, and further studies are certainly needed in this field.

Keywords: breast cancer, lobular cancer, pleomorphic, mastectomy

\section{Introduction}

Invasive pleomorphic lobular carcinoma (P-ILC) was first described in 1982 by Dixon et $\mathrm{al}^{1}$ and in 1987 by Page et $\mathrm{al}^{2}{ }^{2}$ and represents $\sim 2 \%-4 \%$ of invasive carcinomas in two different series. ${ }^{3}$

P-ILC is associated with worse prognostic factors (i.e., larger tumor size, more frequent axillary metastasis) compared with other lobular carcinomas (LCs), and this explains the higher risk of recurrence and decreased survival rate often reported in literature, although in some reports, no differences are noted. ${ }^{1,4,5,6-9}$ Fundamental histologic characteristics of P-ILC are represented by a growth pattern typical of lobular cancer (Figure 1A) and loss of E-cadherin (Figure 1B), also exhibiting a high degree of atypia and cellular pleomorphism, hyperchromatic nuclei (Figure 1C) and a higher mitotic index than classic-ILC (C-ILC). It is often associated with a pleomorphic "in situ" component with comedonecrosis (Figure 1D). It can also present an apocrine or histiocytoid differentiation. ${ }^{10}$

The literature reports that although invasive lobular carcinoma (ILC) is treated with mastectomy more often when compared with infiltrating ductal carcinoma (IDC), 


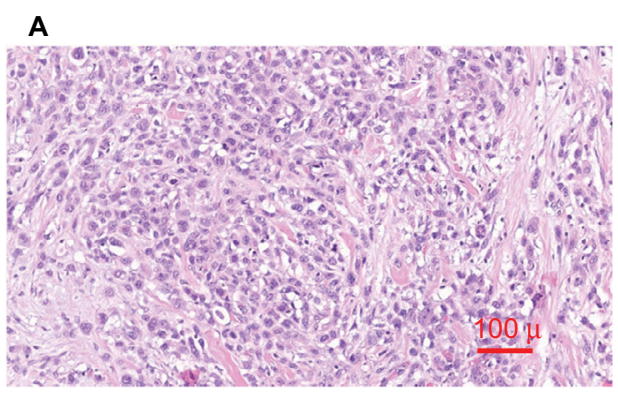

B

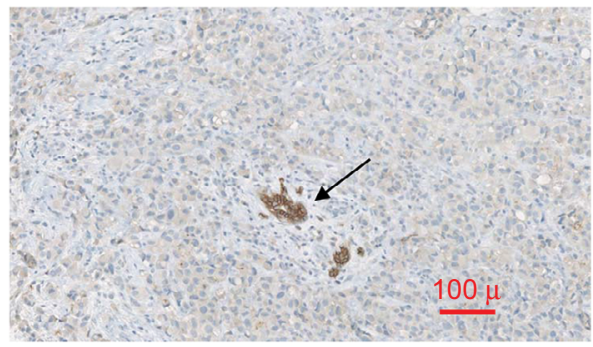

D
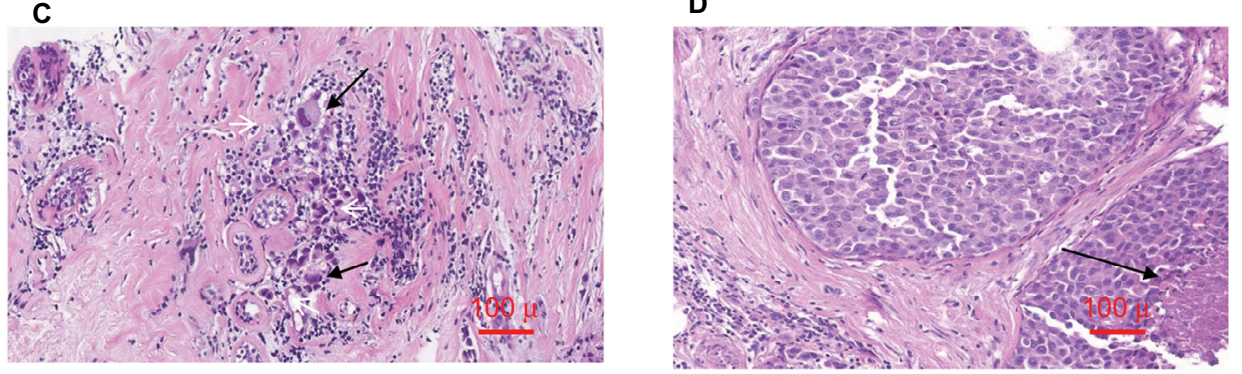

E

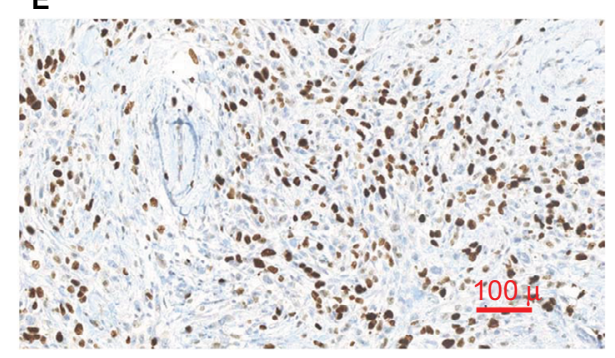

G

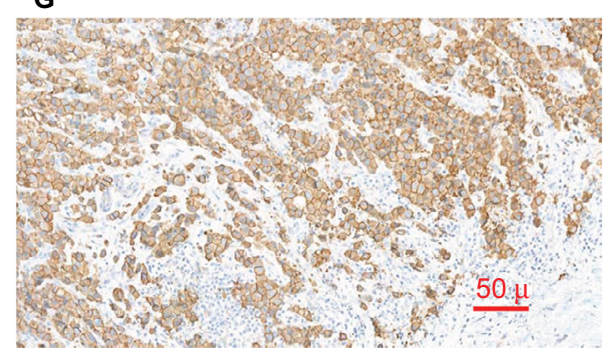

$\mathbf{F}$

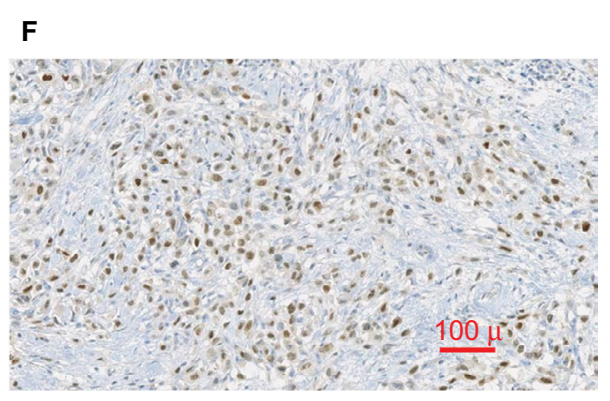

H

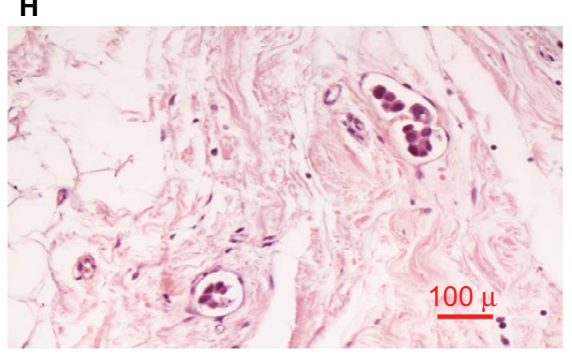

Figure I (A) H-E staining. P-ILC shows the same growth pattern as C-ILC. (B) Low expression or negativity for E-cadherin. The arrow indicates residual ductal structures. (C) H-E staining. Pleomorphic histiocytoid cells with nuclear hyperchromasia (arrows). (D) H-E staining. Comedonecrosis (arrow - in situ component). (E) High Ki67 in P-CLI. (F) neu overexpression (score 3+). (G) p53 overexpression, with weak positivity in more than $50 \%$ of cells (score 2 ). (H) H-E staining. LVI. A to G magnification $\times 20, \mathbf{H}$ magnification $\times 40$.

Abbreviations: H-E, hematoxylin-eosin; LVI, lymphovascular invasion; P-ILC, pleomorphic invasive lobular carcinoma; C-ILC, classic invasive lobular carcinoma.

survival and risk of local recurrence are similar, and therefore, the debate regarding surgical options for patients with lobular cancer is of particular interest. ${ }^{11}$

Although P-ILC and high-grade IDC (HG-IDC) are two distinct pathologic entities, they share similarities regarding nuclear grade, biomolecular and clinical characteristics.

The purpose of our study is to retrospectively analyze our experience with P-ILC and to compare its clinicopathologic features and surgical treatment with those of C-ILC and HG-IDC.

\section{Materials and methods}

From January 2010 to June 2015, 1542 patients with primary breast cancer were treated at our Breast Unit. Recurrent cases or cases after neoadjuvant chemotherapy were excluded. Mixed cases (C-ILC with pleomorphic or IDC areas) were also excluded.

Data available for each case were age, stage according to International Union against Cancer and the American Joint Committee on Cancer (UICC/AJCC) 2010, histologic grade (G) according to the modified Scarff, Bloom and Richardson 
score (Nottingham Combined Histologic Grade), multifocality (m), lymphovascular invasion (LVI) and type of surgery (S). ${ }^{12,13}$ If LVI was suspected, immunohistochemistry (IHC) with CD31 antibody was evaluated to distinguish it from artifacts.

We performed IHC for estrogen receptor (ER), progesterone receptor (PR), proliferative index (Ki67), p53 expression (p53) and HER2/neu oncogene expression. In cases of doubtful histotype, IHC for E-cadherin was performed. The clones used are reported in Table 1.

p53 was evaluated semi-quantitatively by using a scoring system as shown in Table 2.

HER2/neu was evaluated according to American Society of Clinical Oncology, College of American Pathologists (ASCO/CAP) 2013 guidelines, and cases prior to 2013 were re-evaluated. Cases scored $2+$ were evaluated with dualcolor fluorescence in situ hybridization on the centromere of chromosome 17 and on oncogene HER2/neu (locus 17q12, Kreatech $^{\mathrm{TM}}$ ) according to ASCO/CAP 2013. ${ }^{14}$

$\mathrm{P}$-ILC was defined as a high nuclear grade malignancy (significant cytologic atypia and pleomorphism) with lobular characteristics (loosely cohesive cells and negativity for E-cadherin), while HG-IDC was defined as IDC with a Scarff, Bloom and Richardson modified score (Nottingham Combined Histologic Grade) superior to $7 .{ }^{13}$

All treatments were discussed at the multidisciplinary weekly conference, and decisions regarding treatments verbalized in a standard format.

We recorded the type of surgery (mastectomy or partial resection, with or without removal of the nipple-areola complex [NAC]).

We also examined the quantity and type of neoplastic involvement of the NAC. When removed, the NAC was completely sectioned into 3-4 mm thick slices and histologically examined.

Table I Immunohistochemistry

\begin{tabular}{lll}
\hline & Clone & Source \\
\hline ER & SPI & Roche $^{\mathrm{TM}}$ \\
$\mathrm{PR}$ & IE2 & Roche \\
$\mathrm{Ki67}$ & $30-9$ & Roche \\
P53 & DO-7 & Roche \\
HER2/neu & CBII & Cell Marque ${ }^{\mathrm{TM}}$ \\
CD31 & JC70 & Cell Marque \\
E-cadherin & 36 & Roche \\
\hline
\end{tabular}

Abbreviations: ER, estrogen receptor; PR, progesterone receptor.
Two pathologists reviewed the histologic material independently (LC and DC). Assessment of tumor type was performed according to the criteria outlined in the World Health Organization Classification of Tumors. ${ }^{15}$

Statistical analysis was performed with a statistical package. Student's $t$-test for significance between means and binomial analyses using Pearson's $\chi^{2}$ test were performed.

The study was conducted according to the principles laid down in the Declaration of Helsinki. The protocol was reviewed and approved by the Institutional Review Board of the San Giovanni-Addolorata Hospital, and we received a waiver for obtaining patient consent to review their cases, as data were analyzed in aggregate.

\section{Results}

Clinicopathologic data are presented in Table 3 . There were 40 cases of P-ILC, while 126 were defined as C-ILC and 574 as HG-IDC.

The median age and tumor size were not significantly different in the three groups.

Both P-ILC and C-ILC were more frequently multicentric or multifocal than HG-IDC.

Lymph node metastases were more frequently diagnosed in P-ILC compared to C-ILC $(P<0.05)$, and more than three lymph node metastases were more frequently diagnosed in P-ILC (20\%) than in C-ILC $(11 \% ; P<0.05)$.

Hormone receptor positivity was more frequently expressed in P-ILC and C-ILC compared to HG-IDC $(P<0.01)$, and differences in average ER/PR value between P-ILC and C-ILC were noted.

While C-ILC was never HER2/neu+, 20\% of HG-IDC and $10 \%$ of $\mathrm{P}$-ILC were amplified $(P<0.01)$. Absolute values of Ki67 were higher for HG-IDC and lower for C-ILC, while P-ILC showed intermediate results $(P<0.01)$.

Similar findings were registered for LVI positivity $(P<0.01)$.

p53 was rarely positive in C-ILC. In P-ILC, it was weakly to moderately positive in $17.5 \%$ of cases (Figure $1 \mathrm{~F}$ ), while it was variably positive, from weak to strong, in $41.6 \%$ of cases of HG-IDC. These results were statistically significant $(P<0.01)$.

Incidence of mastectomy or breast conservation did not differ among the three groups (Table 4). The NAC was

Table 2 p53 semi-quantitative score

\begin{tabular}{ll}
\hline Score $\mathbf{0}$ & Positivity in less than $10 \%$ of cells \\
Score I & Weak positivity in more than $10 \%$ and less than $50 \%$ of cells \\
Score 2 & Strong positivity in more than $10 \%$ and less than $50 \%$ of cells or weak positivity in more than $50 \%$ of cells \\
Score 3 & Strong positivity in more than $50 \%$ of cells \\
\hline
\end{tabular}


Table 3 Clinicopathologic characteristics

\begin{tabular}{|c|c|c|c|c|c|c|}
\hline & \multirow[t]{2}{*}{ P-ILC $(n=40), \%$} & \multirow[t]{2}{*}{ C-ILC $(n=I 26), \%$} & \multirow[t]{2}{*}{ HG-IDC $(n=574), \%$} & \multicolumn{3}{|l|}{$P$-value } \\
\hline & & & & P-ILC/HG-IDC & P-ILC/C-ILC & C-ILC/HG-IDC \\
\hline Age (years), median & 66 & 64 & 63 & NS & NS & NS \\
\hline Multifocality & II (27.5) & $38(30)$ & $96(17)$ & $<0.01$ & NS & $<0.01$ \\
\hline \multicolumn{7}{|l|}{ Size $(\mathrm{cm})$} \\
\hline$<2$ & $24(60)$ & $82(65)$ & $35 I(6 I)$ & & & \\
\hline$>2$ & $16(40)$ & $44(35)$ & $223(39)$ & NS & NS & NS \\
\hline \multicolumn{7}{|l|}{ Nodal status } \\
\hline Negative & $23(57.5)$ & $86(68)$ & $35 I(6 I)$ & NS & NS & 0.1 \\
\hline Positive & $17(42.5)$ & $40(32)$ & $223(39)$ & NS & $<0.05$ & $<0.05$ \\
\hline$>3$ Lymph nodes & $8(20)$ & $14(1 \mathrm{I})$ & $95(16.5)$ & NS & $<0.05$ & $<0.05$ \\
\hline $\mathrm{ER}+$ & $38(95)$ & $126(100)$ & 431 (75) & $<0.01$ & NS & $<0.01$ \\
\hline Average ER value & $92 \pm 25$ & $98 \pm 10$ & $72 \pm 43$ & $<0.01$ & $<0.05$ & $<0.01$ \\
\hline $\mathrm{PR}+$ & $30(75)$ & $224(75)$ & $362(63)$ & $<0.05$ & NS & $<0.05$ \\
\hline Average $P R$ value & $57 \pm 38$ & $58 \pm 39$ & $42 \pm 40.5$ & $<0.05$ & NS & $<0.05$ \\
\hline Ki67 average value & $27 \pm 15$ & $10 \pm 6$ & $39 \pm 22$ & $<0.01$ & $<0.01$ & $<0.01$ \\
\hline \multicolumn{7}{|l|}{ p53 } \\
\hline Score 0 & $33(82.5)$ & 118 (94) & $335(58)$ & $<0.01$ & $<0.01$ & $<0.01$ \\
\hline Score I+ & $6(15)$ & $8(6)$ & $97(17)$ & & & \\
\hline Score 2+ & I (2.5) & 0 & $54(9)$ & & & \\
\hline Score 3+ & 0 & 0 & $88(15)$ & & & \\
\hline HER2/neu & $4(10)$ & 0 & II 4 (20) & $<0.01$ & $<0.01$ & $<0.01$ \\
\hline $\mathrm{LVI+}$ & $4(10)$ & $3(2)$ & $129(22.5)$ & $<0.01$ & $<0.01$ & $<0.01$ \\
\hline
\end{tabular}

Abbreviations: C-ILC, classic invasive lobular carcinoma; ER, estrogen receptor; HG-IDC, high-grade invasive ductal carcinoma; LVI, lymphovascular invasion; NS, nonsignificant; P-ILC, pleomorphic invasive lobular carcinoma; PR, progesterone receptor.

Table 4 Surgical treatment

\begin{tabular}{lllll}
\hline & P-ILC (\%) & C-ILC (\%) & HG-IDC (\%) & P-value \\
\hline Mastectomy & $10(25)$ & $41(32.5)$ & $153(26.6)$ & NS \\
Partial resection & $30(75)$ & $85(67.5)$ & $421(73.4)$ & NS \\
\hline
\end{tabular}

Abbreviations: C-ILC, classic invasive lobular carcinoma; HG-IDC, high-grade invasive ductal carcinoma; NS, nonsignificant; P-ILC, pleomorphic invasive lobular carcinoma.

removed in 175 cases (23.6\%); in the remaining cases, a nipple-sparing approach was adopted, with no differences noted among the three groups. NAC more frequently involved in lobular cases rather than in HG-IDC cases $(P<0.05)$. However, the type of involvement was different, as the NAC involved direct infiltration of the tumor or cancer in situ (pagetoid spreading) in the former and lymphovascular invasion of the nipple in the latter (Table 5).

\section{Discussion}

To our knowledge, this is the first clinicopathologic study comparing P-ILC with C-ILC and HG-IDC. Our experience shows that P-ILC is rare, representing $\sim 3 \%$ of all breast cancer cases. While P-ILC shares some features with each of the other two histologic forms, it represents an uncommon, autonomous entity.

P-ILC is a high-degree malignancy of the breast, with frequent lymph node metastases, and often involves more than three lymph nodes, thus differing from C-ILC. However, lymph node positivity and multiple lymph node involvement were similar when P-ILC was compared to HG-IDC. P-ILC rarely does not express ER positivity, and this may be explained by an apocrine differentiation in some of these cases. Similarly, there was a different PR expression between LCs (classic and pleomorphic) and HG-IDC, while no difference was found between C-ILC and P-ILC.

Ki67 in P-ILC was intermediate between those of C-ILC (usually very low, below 15\%) and HG-IDC (usually high; in any case $>20 \%$, and frequently as high as $90 \%$ ), as shown in Figure 1E.

While HER2/neu was amplified in $20 \%$ of HG-IDC, it was never amplified in C-ILC and it was positive in $10 \%$ of P-ILC. This is similar to a previous report. ${ }^{16}$ The latter may be associated with a histiocytoid variant, previously described with low expression of hormone receptors, as we reported in our experience. ${ }^{17-19}$

p53 was rarely and weakly expressed in C-ILC, while it was positive in $17.5 \%$ of cases of P-ILC (Figure $1 \mathrm{G}$ ) and in over $40 \%$ of cases of HG-IDC.

We confirm that peritumoral LVI was rare in C-ILC, and it occurred in $10 \%$ of P-ILC and $22.5 \%$ of HG-IDC (Figure 1H). The cells of LC are poorly cohesive and often found in chains within "slit-like" spaces not covered by 
Table 5 Involvement of NAC when removed

\begin{tabular}{lllll}
\hline Type of involvement & P-ILC $(\mathbf{n}=\mathbf{I 0}), \%$ & C-CLI $(\mathbf{n}=\mathbf{3} \mathbf{I}), \%$ & HG-IDC $(\mathbf{n}=\mathbf{I 3 4}), \%$ & $\mathbf{P}$-value \\
\hline Any & $6(60)$ & $18(58)$ & $5 \mathrm{I}(38)$ & 0.06 \\
Carcinoma in situ & $\mathrm{I}(10)$ & $3(10)$ & $21(16)$ & 0.04 \\
Stromal infiltration & $5(50)$ & $15(48)$ & $23(17)$ & 0.9 \\
Lymphatic spread & 0 & 0 & $7(5)$ & 0.04 \\
\hline
\end{tabular}

Abbreviations: NAC, nipple-areola complex; C-ILC, classic invasive lobular carcinoma; HG-IDC, high-grade invasive ductal carcinoma; P-ILC, pleomorphic invasive lobular carcinoma.

endothelium (CD34+/CD31+). These areas, known as "prelymphatic spaces", are lined by cells CD31-/CD34+. ${ }^{20}$ This network allows quick communication between the stroma and the main lymphatic system. The real invasion of lymphovascular space (CD31+) was present in only $4.2 \%$ of all ILC cases, while it was $10 \%$ for P-ILC and $22.5 \%$ for HG-IDC.

Due to overlapping morphologic and IHC characteristics between C-ILC and HG-IDC, the origin of P-ILC has been debated. Some authors have suggested that P-ILC is a highgrade IDC with loss of E-cadherin expression. ${ }^{21,22}$ However, molecular studies have demonstrated that P-ILC and C-ILC share several molecular features such as inactivation or downregulation of E-cadherin and a cell-to-cell adhesion molecule, as well as recurrent genomic changes. Comparative genomic hybridization showed a gain of $1 \mathrm{q}(1 \mathrm{q}+)$ in $100 \%$ and a loss of $16 \mathrm{q}\left(16 \mathrm{q}^{-}\right)$in $93 \%$ of cases, features that are typical of LCs, but are not seen in HG-IDC. Fluorescence in situ hybridization analysis showed $\operatorname{der}(1 ; 16) / \operatorname{der}(16) \mathrm{t}(1 ; 16)$ rearrangement, a typical "lobular phenotype". P-ILC may have the same precursor and the same genetic pathway as C-ILC. ${ }^{22,23}$ Additionally, it has been argued that P-ILC may develop through a molecular pathway similar to that of C-ILC, with the acquisition of further molecular alterations, more typical of high-grade ductal carcinomas, such as gain of HER2/neu, c-myc, p53 positivity and amplification of 8q24, $12 q 13$ and $20 q 13 .{ }^{6,21-24}$ So far, there have been no reported studies to establish the frequency of p53 and HER2/neu overexpression in P-ILC.

Radiologic features of LC can vary, as tumors are most commonly detected as a spiculated mass with or without calcifications on mammography or ultrasonography, but C-ILC and P-ILC cannot be differentiated on the bases of imaging findings. One report describes a false-negative rate with mammography in $4.5 \%$ of P-ILC and $14.9 \%$ of C-ILC. ${ }^{25}$ In the same study, magnetic resonance imaging revealed additional foci in five of 13 P-ILCs (38\%) and 18 of 40 C-ILCs (45\%), but was similar to ultrasound.

Sparse data exist in literature regarding the clinical presentation, prognosis and surgical treatment of P-ILC compared with C-ILC and HG-IDC. Availability of limited data is due to the relative rarity of P-ILC, and studies usually have small sample sizes. It has been recently emphasized that patients with invasive LC, although presenting more frequently with multifocal and multicentric disease, have similar survival and local control rates compared with invasive ductal carcinoma. ${ }^{11}$ Similar findings have been described by a retrospective analysis of 1650 patients comparing these two groups, even when only patients treated with breast conservation were analyzed. ${ }^{26}$ When compared to C-ILC, it is reported that P-ILC is diagnosed at a more advanced stage, the tumor diameter can be larger, lymph node metastases are more frequently noted and that overall survival is shorter, with more frequent occurrence of distant metastases. ${ }^{1,5,27}$ However, similar to our findings, there was no significant difference in the median patient age. ${ }^{6}$

\section{Conclusion}

Our study has several limitations, as it is retrospective in nature and involves a small number of cases. However, it is evident in our experience that P-ILC is an uncommon autonomous entity that shares some features with both C-ILC and HG-IDC. While hormone receptors are almost always positive, HER2/neu is amplified in $10 \%$ of cases. Suggested surgical treatment does not differ in our experience, but lymph nodes are more often involved in P-ILC and multiple metastases are encountered in some $20 \%$ of patients.

These findings may be useful in understanding this clinical entity, and further studies are certainly needed in order to clarify the clinical options and strategies.

\section{Acknowledgment}

This work was supported in part by Fondazione Prometeus, ONLUS.

\section{Disclosure}

The authors report no conflicts of interest in this work.

\section{References}

1. Dixon JM, Anderson TJ, Page DL, Lee D, Duffy SW. Infiltrating lobular carcinoma of the breast. Histopathol. 1992;6(2):149-161.

2. Page DL, Anderson TJ, Sakamoto G. Infiltrating carcinoma: major histological types. 1987, WB Saunders, London. 
3. Riva C, Dainese E, Caprara G, et al. Immunohistochemical study of androgen receptors in breast carcinoma. Evidence of their frequent expression in lobular carcinoma. Virchows Arch. 2005;447(4):695-700.

4. Eusebi V, Magalhaes F, Azzopardi JC. Pleomorphic lobular carcinoma of the breast: an aggressive tumor showing apocrine differentiation. Hum Pathol. 1992;23(6):655-662.

5. Liu YL, Choi C, Lee SM, et al. Invasive lobular breast carcinoma: pleomorphic versus classical subtype, associations and prognosis. Clin Breast Cancer. Epub 2017 Jun 23.

6. Buchanan CL, Flynn, LW, Murray MP, et al. Is pleomorphic lobular carcinoma really a distinct clinical entity? J Surg Oncol. 2008;98(5):314-317.

7. Di Costanzo D, Rosen PP, Gareen I, Franklin S, Lesser M. Prognosis in infiltrating lobular carcinoma. An analysis of "classical" and variant tumor. Am J Surg Pathol. 1990;14(1):12-23.

8. Sahin S, Karatas F, Erdem GU, Hacioglu B, Altundag K. Invasive pleomorphic lobular histology is an adverse prognostic factor on survival in patients with breast cancer. Am Surg. 2017;83(4):359-364.

9. Orvieto E, Maiorano E, Bottiglieri L, et al. Clinicopathologic characteristics of invasive lobular carcinoma of the breast: results of an analysis of 530 cases from a single institution. Cancer. 2008;113(7):1511-1520.

10. Waltord N, ten Velden J. Histiocytoid breast carcinoma: an apocrine variant of lobular carcinoma. Histopathology. 1989;14(5):515-522.

11. Fortunato L, Mascaro A, Poggia I, et al. Lobular breast cancer: same survival and local control compared with ductal cancer, but should both be treated the same way? Analysis of an institutional database over a 10-years period. Ann Surg Oncol. 2012;19(4):1107-1114.

12. AJCC. AJCC Cancer Staging Manual, 8th Ed., 2017. Springer.

13. Elston CW, Ellis IO. Pathological prognostic factors in breast cancer. I. The value of histological grade in breast cancer: experience from a large study with long-term follow-up. Histopathology. 1991;19(5):403-410.

14. Wolff AC, Hammond ME, Hicks DG et al; College of American Pathologists. Recommendations for human epidermal growth factor receptor 2 testing in breast cancer. American Society of Clinical Oncology/ College of American Pathologists clinical practice guideline update. J Clin Oncol. 2013;31(31):3997-4013.

15. World Health Organization. WHO Classification of Tumours of the Breast. IARC Press, 2012.

16. Lien HC, Chen YL, Juang YL, Jeng TM. Frequent alterations of HER2 through mutation, amplification, or overexpression in pleomorphic lobular carcinoma of the breast. Breast Cancer Res Treat. 2015;150(2):447-455.
17. Hoff ER, Tubbs RR, Myles JL, Procop GW. Her2/neu amplification in breast cancer: stratification by tumor type and grade. Am J Clin Pathol. 2002;117(6):916-921.

18. . Frolik D, Caduff R, Varga Z. Pleomorphic lobular carcinoma of the breast: its cell kinetics, expression of oncogenes and tumour suppressor genes compared with invasive ductal carcinomas and classical infiltrating lobular carcinomas. Histopathology. 2001;39(5): 503-513.

19. Middleton LP, Palacios DM, Bryant BR, Krebs P, Otis CN, Merino MJ. Pleomorphic lobular carcinoma: morphology, immunohistochemistry, and molecular analysis. Am J Surg Pathol. 2000;24(12): 1650-1656.

20. Hartveit F. Attenuated cells in breast stroma: the missing lymphatic system of the breast. Histopathology. 1990;16(6):533-543.

21. Reis-Filho JS, Simpson PT, Jones C, et al. Pleomorphic lobular carcinoma of the breast: role of comprehensive molecular pathology in characterization of an entity. J Pathol. 2005;207(1):1-13.

22. Simpson PT, Reis-Filho JS, Lambros MB, et al. Molecular profiling pleomorphic lobular carcinomas of the breast: evidence for a common moleculargenetic pathway with classic lobular carcinomas. J Pathol. 2008;215(3):231-244.

23. Vargas AC, Lakhani SR, Simpson PT. Pleomorphic lobular carcinoma of the breast: molecular pathology and clinical impact. Future Oncol. 2009;5(2):233-243.

24. Palacios J, Sarrio D, Garcia-Macias MC, Bryant B, Sobel ME, Merino MJ. Frequent E-cadherin gene inactivation by loss of heterozygosity in pleomorphic lobular carcinoma of the breast. Mod Pathol. 2003;16(7):674-678.

25. Jung HN, Shin JH, Han BK, Ko EY, Cho EY. Are the imaging features of the pleomorphic variant of invasive lobular carcinoma different from classic ILC of the breast? Breast. 2013;22(3):324-329.

26. Biglia N, Maggiorotto F, Liberale V, et al. Clinical-pathologic features, long term-outcome and surgical treatment in a large series of patients with invasive lobular carcinoma (ILC) and invasivecarcinoma (IDC). Eur J Surg Oncol. 2013;39(5):455-460.

27. Bentz JS, Yassa N, Clayton F. Pleomorphic lobular carcinoma of the breast: clinicopathologic features of 12 cases. Mod Pathol. 1998;11(9):814-822.
Breast Cancer - Targets and Therapy

\section{Publish your work in this journal}

Breast Cancer - Targets and Therapy is an international, peerreviewed open access journal focusing on breast cancer research, identification of therapeutic targets and the optimal use of preventative and integrated treatment interventions to achieve improved outcomes, enhanced survival and quality of life for the cancer patient.

\section{Dovepress}

The manuscript management system is completely online and includes a very quick and fair peer-review system, which is all easy to use. Visit http://www.dovepress.com/testimonials.php to read real quotes from published authors. 